\title{
Student Health Clinic
}

National Cancer Institute

\section{Source}

National Cancer Institute. Student Health Clinic. NCI Thesaurus. Code C114862.

An onsite healthcare facility that provides routine therapeutic and preventive services to students. 